\title{
INFERENCE FOR THE EXTENT PARAMETER OF DAMAGE BY TSUNAMI WITH POINCARÉ CONES
}

\author{
Toshimasa Nagai* and Toshinari Kamakura ${ }^{\dagger}$
}

\begin{abstract}
The cone-convex hull by complement (ccc-hull) is a generalized convex hull created from Poincaré Cones. We propose a new approach with the ccc-hull for the simulation of the extent of damage by a tsunami, and simulate the damaged area at the time of the Great East Japan Earthquake for approximate damage by samples from a twodimensional Non-homogeneous Poisson Process. Then, we consider the problem of the estimation of the parameter $\rho$, which corresponds to the opening angle of the Poincaré Cones. We believe our suggestion can be used to predict the extent of a tsunami in a specified area in Japan.
\end{abstract}

\section{Introduction}

Recently we have suffered from the damage caused by some big natural disasters such as earthquakes, tsunamis, river floods and landslides. Among recent natural disasters in Japan, the Great East Japan Earthquake caused the greatest human and economic damage in Japanese recorded history. The maximum magnitude of this earthquake was 9.0. The Fire and Disaster Management Agency of Japan reported that the number of deaths including the earthquake disaster-related deaths was 19,418 people and the number of missing people reached 2,592. In addition, the Metropolitan Police Department of Japan reported that the cause of death of approximately $90 \%$ of 15,786 human remains found after the disaster was the tsunami. Most of those who drowned failed to evacuate because the tsunami was unexpectedly large. The Japan Meteorological Agency and the Cabinet Office, government of Japan, reported that the wave height of this tsunami reached more than $10 \mathrm{~m}$ and the runup height reached nearly $40 \mathrm{~m}$ in some areas. For the prevention or reduction of such damage, it is important to predict its extent in detail. According to the geographical characteristics, from prediction and visualization of the extent of damage by quantifying the scale of the natural disaster, we are able to perceive the risk and expect to evacuate appropriately in time of the disaster.

As for the inference of the extent of damage, there are methods such as modeling a physical phenomenon of the natural disaster mathematically and simulating with experimental equipment. For numerical simulation of tsunami generation or propagation, hydrodynamic approaches such as long-wave theory and ray theory are generally used. Details of these methods were given by Satake (1991). The tsunami hydrodynamic load is a piece of experimental equipment that can reconstruct the movement of a tsunami on a large scale. Kihara et al. (2014) confirmed characteristics of the wave pressure of a tsunami on a tide wall using

\footnotetext{
*Graduate School of Science and Engineering, Chuo University, Kasuga, Bunkyo-ku, Tokyo, 112-8551, Japan E-mail: a11.cka6@g.chuo-u.ac.jp

${ }^{\dagger}$ Faculty of Science and Engineering, Chuo University, 1-13-27 Kasuga, Bunkyo-ku, 112-8551, Japan

Key words: Cone-convex hull by complement; Poincaré Cone; Two-dimensional Non-homogeneous Poisson Process
} 
this device. Sato et al. $(2014,2016)$ assumed a generalized Pareto distribution for the wave height of the tsunami, and estimated its parameters from past run-up events in each of a set of $1 \mathrm{~km}$ grid squares (called a 'mesh'). They also estimated the frequency of the run-up on each mesh. A tsunami due to the occurrence of an earthquake is closely related to the slip-deficit for the interplate near the trench. Yokota et al. (2016) estimated an interplate slip-deficit rate distribution on the Nankai Trough subduction zone using seafloor geodetic data. Seafloor geodetic data was observed from seafloor sensors by GPS and acoustic measurements. They confirmed regions of high slip-deficit rates which is likely to cause the tsunami. However, the mathematical model of the disaster depends on many physical restrictions. For instance, in the case of a tsunami, it will be necessary to think about some factors such as a wave, wind, topography and a sea area. In addition, the simulation with the large-scale experimental equipment has a problem that expenses will run up. From such a background, in this study, we think about a new approach using a computational geometry-like technique as a method to estimate damage extents of the disaster. Specifically, we deal with a generalized convex hull. Our simulation model does not need to use the governing equation and experimental equipment like conventional methods. Therefore, our model has the advantage that it does not depend on physical restrictions and it is not costly.

A convex hull is the smallest convex polygon or convex polyhedron that contains all given points. In computational geometry, for instance, the convex hull has important applications in pattern recognition, cluster analysis and image processing, among others (PateiroLópez and Rodríguez-Casal, 2010). In addition, the convex hull is also applied in the set estimation that reconstructs a set from a finite set of points taken into it, but it is not suitable for the estimation of non-convex sets. For that problem, a generalized convex hull is often used. There are such examples as the $\alpha$-convex hull and the cone-convex hull by complement (ccc-hull). The $\alpha$-convex hull can be expressed by the intersection of a family of complements of balls with radius $\alpha>0$ and allows for considering a set with inward peaks and holes which the normal convex hull is not able to estimate depending on $\alpha$ (PateiroLópez and Rodríguez-Casal, 2010). Cholaquidis et al. (2014) defined the cone-convex that possessed both the cone convexity and the Poincaré cone property $(P C P)$, and they proposed the ccc-hull that is an extension of it. Here, we note that PCP is a regularity condition for sets in the Euclidean space (Cholaquidis et al. , 2014; Mörters and Peres, 2010). The ccchull can be expressed by the intersection of a family of complements of cones with opening angle $\rho \in(0, \pi]$ and radius $h>0$. That is, it is just replacing balls of the $\alpha$-convex hull with cones. Here, in this paper, we call these cones Poincaré Cones. As the envelope of the ccc-hull changes variously depending on the opening angle $\rho$ of Poincaré Cone, it allows us to consider general sets with rougher boundaries much more than the normal convex hull or the $\alpha$-convex hull. In addition, Cholaquidis et al. (2014) say that the reconstruction of a target set from random sample points using these generalized convex hulls entails some loss in generality but, in return, a wealth of valuable results such as the estimation of the boundary and the boundary measure, etc. are typically obtained.

Our purpose is to propose a simulation model of the extent of damage by the tsunami by the new approach with the ccc-hull. For our model, we simulate the extent of damages by the tsunami at the time of the Great East Japan Earthquake in two-dimensions. Then, we adapt the elevation for the area to our model by using sample points from the twodimensional Non-homogeneous Poisson Process. In addition, we consider the selection of the optimal parameter $\rho$ of the ccc-hull for which our model best reconstructs the extent of 
damage. For the reconstruction of the damaged area, we dene the index that expresses the intensity of the tsunami by specifying the relation between the parameter $\rho$ and the sample size $n$ when the ccc-hull is applied. The simulation model consists of three steps. First, the damaged area is estimated by ccc-hull, given the real damaged area. Second, we interpret the angle, which is a parameter of ccc-hull, as the scale of the tsunami. Finally, sample points are used to interpret the run-up of the tsunami.

This paper is organized as follows. In Section 2, we introduce the definition of the ccchull, and describe our simulation model of damage extents of the tsunami. The detail of the generation of sample points with the two-dimensional Non-homogeneous Poisson Process, which expresses the elevation, is given in Section 3. In Section 4, we describe the selection of the optimal parameter that reconstructs the extent of damages the best. Results of the simulation of the extent of damage with the ccc-hull are given in Section 5 and, finally, we conclude with a discussion in Section 6 .

\section{Model}

\subsection{Cone-convex hull by complement}

In the following, we briefly explain the ccc-hull proposed in Cholaquidis et al. (2014) that is a generalized convex hull.

A closed set $S \subset \mathbb{R}^{d}$ is said to be cone-convex by complement with parameters $\rho \in(0, \pi]$, $h>0(\rho, h-c c c)$ for all $y \in \partial S$ if and only if

$$
S=\bigcap_{\left\{y: C_{\rho, h}(y) \cap S=\emptyset\right\}}\left\{C_{\rho, h}(y)\right\}^{c},
$$

where $C_{\rho, h}(y)$ denotes a finite cone with vertex $y$, of type $C_{\rho, h}(y)=C_{\rho}(y) \cap B(y, h)\left(C_{\rho}(y)\right.$ and $B(y, h)$ are the open cone with opening angle $\rho$ and vertex $y$, and the open ball with center $y$ and radius $h$, respectively). In this paper, we call $C_{\rho, h}(y)$ a Poincaré Cone. Generally, if $c x \in S$ for any positive scalars $c$, and any $x \in S$, a set $S$ is a cone. Although such a cone has a region that is infinite in extent, Poincaré Cones are finite cones that are separated by a radius $h$.

A closed set $S$ is expressed as the intersection of a family of complements of cones if it is the $\rho, h$-ccc. Then, the domain that appears as the polygon defined by Poincaré Cones is called a ccc-hull. Figure 1 shows the ccc-hull of complete spatial randomness (CSR) points in a hexagram. The CSR is the case such that the occurring of points is determined by the two-dimensional homogeneous Poisson Process. See also Voss (2013) about the homogeneous Poisson Process. Here, parameters $\rho$ and $h$ are the angle and radius of Poincaré Cones, respectively. We can see that the hexagram is well generated by the ccc-hull. The algorithm of the ccc-hull for the two-dimensional case was proposed by Cholaquidis et al. (2014). The $\mathrm{R}$ code for it can be downloaded from http://www.uam . es/antonio.cuevas/exp/ccc-algorithm.txt. See Cholaquidis et al. (2014) about some numerical results for this algorithm. However, the program is very slow and we improved the program by parallelizations using the $\mathrm{R}$ package foreach; the improved version is open to access by http://www.indsys.chuo-u.ac.jp/ kmlab/repo/. In addition, we ran a benchmark test of these programs. For the details of this benchmarking, refer to Section 7 . Note that all ccc-hulls in our simulation are obtained by our package. 


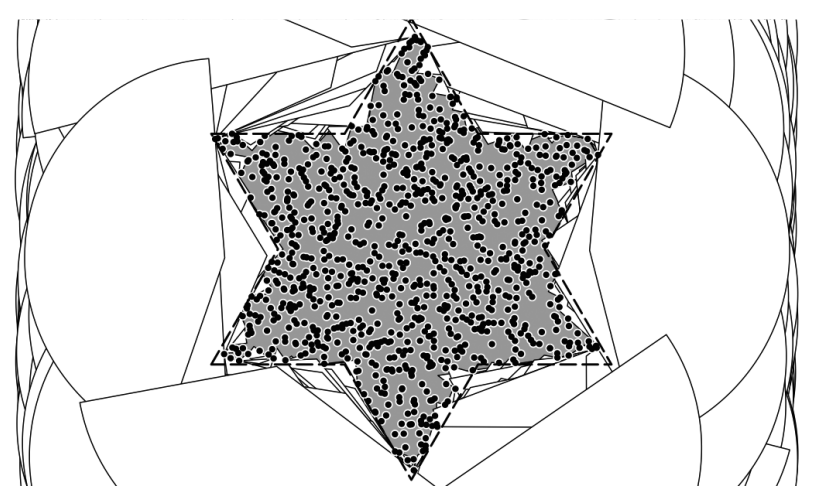

Fig. 1: The ccc-hull with parameters $\rho=\pi / 4, h=1 / 2$ (shaded area) constructed from 1200 CSR points of a hexagram. Parameters $\rho$ and $h$ are the angle and radius of Poincaré Cones, respectively.

\subsection{Simulation model of the extent of damage}

In this paper, we consider the new approach with the ccc-hull for the simulation of the extent of damage by a tsunami. Then, the extent of damage that is estimated indicates the visualized two-dimensional region like an aerial photograph and a satellite photograph.

The extent of damage is simulated by following procedures.

1. Sample $n$ points from the area in which the tsunami occurred.

2. Apply the ccc-hull with the parameter $\rho$ for $n$ sample points.

Here, we assume that the ccc-hull is the area not damaged by the tsunami, i.e., the estimated safe area, and the Poincaré Cones are the damaged area, i.e., the estimated damage area. In addition, we assume that the sample size $n$ from the damaged area is a disaster prevention power for the tsunami. That is, we interpret that the run-up of the tsunami from a coastline to an inland area becomes large if the sample has high density, and the run-up becomes small if the density is low. This is because of the property of the ccc-hull that the domain where Poincaré Cones can exist becomes narrow if the sample size $n$ is large, that is, the sample has high density, and, conversely, it becomes wider if $n$ is small, that is, the density is low. In this case, a sample point in itself is something that prevents the run-up of the tsunami such as obstacles. From the assumptions and the properties of the sample for the ccc-hull, for example, the following characteristics of a tsunami can be explained. Generally, the tsunami floods and runs amorphously on completely flat land. Then, when the tsunami contacts with obstacles on land, if the obstacles are extensive or compact, such as banks, shore protections or tetrapods, its run-up becomes weaker or stops and it retreats seaward from that point. On the other hand, for scattered obstacles such as cars or buildings, it runs around and overflows. Characteristics of a tsunami with respect to the run-up and the flood like this correspond to the property of the ccc-hull in relation to sample size. In addition, we assume that the parameter $\rho$ is the scale of the tsunami. That is, we interpret that the run-up of the tsunami from a coastline to an inland area becomes small if the parameter $\rho$ is close to $\pi$, and the run-up becomes large if $\rho$ is close to a zero. This is also because of the property of the ccc-hull that the boundary of the set can be expressed in detail if 
the opening angles of the Poincaré Cones $\rho$ are small, and, conversely, it is expressed more smoothly if $\rho$ is large. The power of the run-up depends on the wave height of the tsunami. If we do not consider the influence of topography and obstacles, the run-up of a tsunami with high wave height is large and the run-up with a low wave height is small. Since wave height is related to the scale of a tsunami, from the property of the ccc-hull about the angle, we can explain such characteristics of a tsunami.

Although the location at which points are generated is not restricted if points exist in the area, if possible, it is desirable to place them according to the location information of actual buildings and the like. For example, location information about public facilities and institutions is possible to obtain from the Ministry of Land, Infrastructure, Transport and Tourism (http://nlftp.mlit.go.jp/ksj/index.html). On the other hand, in many cases, location information at the individual housing level is difficult to obtain due to privacy problems and the like. However, if points that prevent the run-up of tsunami are uniformly distributed within the area, the disaster prevention power of each part in the area will be the same. This is a somewhat brute force assumption since it is reasonable to assume that the occurrence of the run-up is different in each part in the area due to buildings and elevations at each point. Therefore, it is necessary to consider how the occurrence of the run-up is reflected in the arrangement of sample points. This will be discussed in Section 3.

For the above assumptions and interpretations, the run-up of the tsunami from a coastline to an inland are is simulated in two-dimensions like Figure 2. It is necessary to set the parameter $\rho$ and the sample size $n$ at any time for our simulation. The selection of the optimal parameter that expresses the extent of damage will be discussed in Section 4.

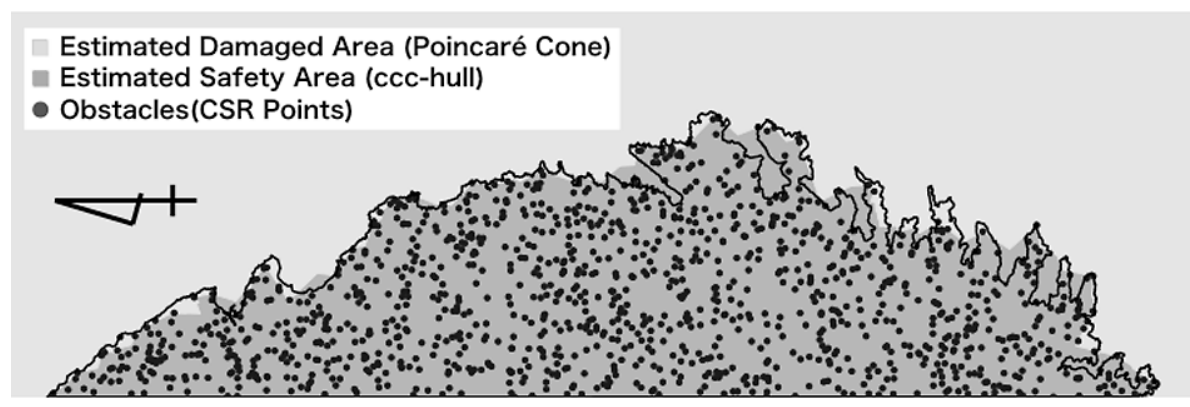

Fig. 2: Simulation of the extent of damage by the ccc-hull with parameters $\rho=5 \pi / 18$ from 1200 CSR points in a part of Iwate.

\section{Modeling of the elevation}

In our study, we simulate and visualize the area of the run-up of the tsunami from a coastline to an inland area by using the ccc-hull and Poincaré Cones. We assume that sample points with the ccc-hull are obstacles to prevent the run-up. Then, the disaster prevention power is the same in each point if we assume the CSR points. However, it is necessary to consider that the height of obstacles influences the level of the run-up. So, in this study, we assume that the disaster prevention power depends on points reached by the tsunami. For this assumption, we consider that points in high elevations are distributed densely, and points in low elevations are distributed sparsely. This means that it is difficult to run-up in 
high elevations and it is easy in low elevations. The density of sample points is expressed by the two-dimensional Non-homogeneous Poisson Process (NHPP). In the following, we describe the generation of the NHPP sample by rejection sampling for the CSR points. See also Christian and Casella (2009) about the rejection sampling.

Let $\lambda(x, y)=z$ be the intensity function that returns the elevation $z$ for the latitude $x$ and longitude $y$ of each point $(x, y)$. In addition, let $\lambda^{*}=\max \lambda(x, y)$ be the maximum value of the intensity function, that is, the maximum value of elevations. Then, for CSR points from the area, we accept ones satisfying conditions such that

$$
U<\frac{\lambda(x, y)}{\lambda^{*}}
$$

where $U$ is a random variable that follows a uniform distribution on the interval $[0,1]$. Samples that are accepted by equation (2) follow a two-dimensional NHPP.

Figure 3 shows the visualization of elevations and NHPP sample points in Iwate. The data of elevations was acquired from National Land Numerical Information download service (http://nlftp.mlit.go.jp/ksj/index.html). We can see that points in high elevations appear dense, and points in low appear sparse.
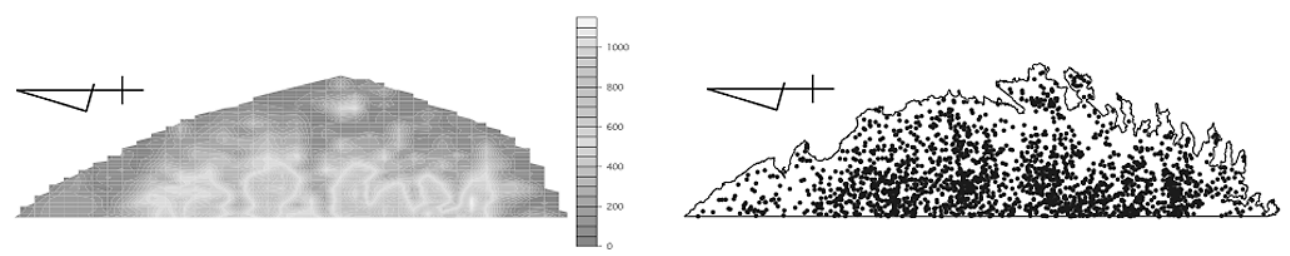

Fig. 3: The visualization of elevations in Iwate (left), and NHPP sample points (right).

\section{Selection of the optimal parameter}

The ccc-hull heavily depends on the parameter $\rho$ and the sample size $n$. However, for the set estimation, we empirically need to decide the parameter $\rho$. For our simulation, we need to give the parameter $\rho$ and the sample size $n$ everytime. Therefore, the selection of a reasonable parameter that expresses the extent of damage is an important problem. If the parameter is not appropriate, for example, the extent of damage is overestimated like the left-hand panel of Figure 4, and the estimated safe area appears to the seaward like the right-hand panel of Figure 4. In this study, for these problems, we discuss the selection of the optimal parameter by obtaining the index with respect to the closeness between the estimated damage area and actual damaged area.
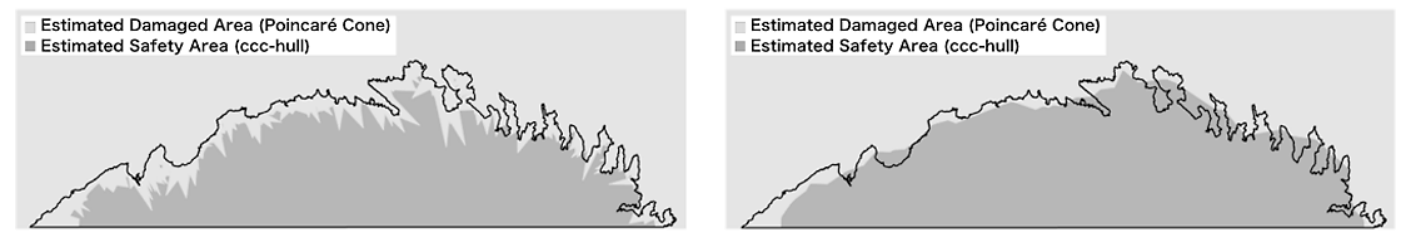

Fig. 4: The overestimation (left), and the underestimation (right) for the simulation of the extent of damage by the ccc-hull to NHPP sample points in Iwate. 


\section{1. $3 \mathrm{D}$ plot}

Given an area where damages are reconstructed by $S_{0}>0$, let $\bar{S}_{0}^{(n)}(\rho)>0$ be the closure set that is spanned by the ccc-hull of $n$ sample points and parameter $\rho$. We say that a value $\rho$ is the best parameter when the following equation (3) holds;

$$
\bar{S}_{0}^{(n)}(\rho) / S_{0}=\left(S_{0}-S_{d}\right) / S_{0},
$$

where $S_{d}>0$ is the actual area that is damaged by tsunami and $S_{0}-S_{d}$ indicates the safe area. In this study, the estimated area expresses the ccc-hull. Then, if the estimated safe area $\bar{S}_{0}^{(n)}(\rho)$ corresponds to the safe area $S_{0}-S_{d}$, we consider that the shape of the ccc-hull approximates the shape of the safe area. Therefore, the area ratio $\bar{S}_{0}^{(n)}(\rho) / S_{0}$ is defined as the index that expresses the closeness between the estimated safe area and the actual safe area as well as the estimated damage area and the actual damaged area. For $\bar{S}_{0}^{(n)}(\rho) / S_{0}=1$, the ccc-hull will generate the original figure including non-convex ones, but we cannot get the damage area. For $\bar{S}_{0}^{(n)}(\rho) / S_{0}>1$, the ccc-hull will generate a larger area than the original figure.

Here, we confirm how the change of the parameter $\rho$ affects the closeness between the estimated damage area and the actual damaged area. Specifically, we consider the 3D plot of the sample size $n$ (x-axis), the parameter $\rho$ (y-axis) and the area ratio $\bar{S}_{0}^{(n)}(\rho) / S_{0}$ (z-axis). The left-hand panel of Figure 5 shows the $3 \mathrm{D}$ plot for the reconstruction of the extent of damage by the tsunami at the time of the Great East Japan Earthquake in Iwate. The 3D plot consists of the three-dimensional scatter diagram of 32,400 values of the area ratio with 180 possible sample sizes $(n=100,200, \ldots, 18000)$ and 180 values of parameter $\rho\left(\rho=1^{\circ}, 2^{\circ}, \ldots, 180^{\circ}\right)$. Then, locations of sample points are given by CSR or NHPP, and directions of cones are given with $\xi \in(0,2 \pi]$. In addition, for radii of cones $h$, we use a large value enough value to exceed the total length of the area corresponding to the estimated damage. The curved surface in the Figure indicates a linear interpolation of this scatter diagram. We see that the ccc-hull is overestimated if the sample size $n$ and the parameter $\rho$ become large, and it is underestimated if $n$ and $\rho$ become small. We show the $x y$-plane that the area ratio becomes $\bar{S}_{0}^{(n)}(\rho) / S_{0}=\left(S_{0}-S_{d}\right) / S_{0}$ in the 3D plot. The extent of damage is reconstructed the best by the sample size $n$ and the parameter $\rho$ when the area ratio $\bar{S}_{0}^{(n)}(\rho) / S_{0}=\left(S_{0}-S_{d}\right) / S_{0}$. This indicates the part of intersection for the curved surface and the $x y$-plane.

\section{2. $\rho$-n curve}

In the cross section that is cut at the area ratio $\bar{S}_{0}^{(n)}(\rho) / S_{0}$ for our 3D plot, the curve that expresses the relation between the parameter $\rho$ and the sample size $n$ appears. In our study, we call this curve the $\rho$ - $n$ curve. The right-hand panel of Figure 5 shows the $\rho$ - $n$ curve when the area ratio is $\bar{S}_{0}^{(n)}(\rho) / S_{0}=\left(S_{0}-S_{d}\right) / S_{0}$ for the 3D plot in the left-hand panel of Figure 5. The $\rho$ - $n$ curve has the tendency that the value of $\rho$ becomes small if the value of $n$ becomes large, conversely, the value of $\rho$ becomes large if the value of $n$ becomes small. In addition, this tendency holds at any value of the area ratio. From the $\rho$ - $n$ curve, generally, for the reconstruction of the domain with the ccc-hull, it is effective that the setting of $\rho$ is large if the sample size $n$ is small, and the setting of $\rho$ is small if the sample size $n$ is large. The $\rho$ - $n$ curve that the area ratio becomes $\bar{S}_{0}^{(n)}(\rho) / S_{0}=\left(S_{0}-S_{d}\right) / S_{0}$ expresses the relation between the parameter $\rho$ and the sample size $n$ for the best reconstruction of the extent of damage by the tsunami. If the ccc-hull is able to be determined for sample points, there exists a combination of $\rho$ and $n$ satisfying $\bar{S}_{0}^{(n)}(\rho) / S_{0}=\left(S_{0}-S_{d}\right) / S_{0}$. 
Here, we explain the selection of the optimal parameter $\rho$, given the locations of sample points, direction of cones and length of $h$. First, we obtain the $\rho-n$ curve so that the area ratio becomes $\bar{S}_{0}^{(n)}(\rho) / S_{0}=\left(S_{0}-S_{d}\right) / S_{0}$ from the $3 \mathrm{D}$ plot. Second, for the obtained curve, we regard $\rho$ as a function of $n$, and substitute $n^{\prime}$ for this function. Finally, we select the value $\hat{\rho}$ that was provided by substituting $n^{\prime}$ as the best parameter that expresses the extent of damage. Then, we note that $\rho$ is optimal under the condition that locations of sample points, directions $\xi$ and radii $h$ are given. In our study, the selected parameter $\hat{\rho}$ indicates the intensity of the tsunami. Any value can be used for $n$ if it is on the support of the $\rho-n$ curve such that the area ratio becomes $\bar{S}_{0}^{(n)}(\rho) / S_{0}=\left(S_{0}-S_{d}\right) / S_{0}$. This is because the ccc-hull is roughly equal to the shape of the actual safe area for such a $\rho$ - $n$ curve.
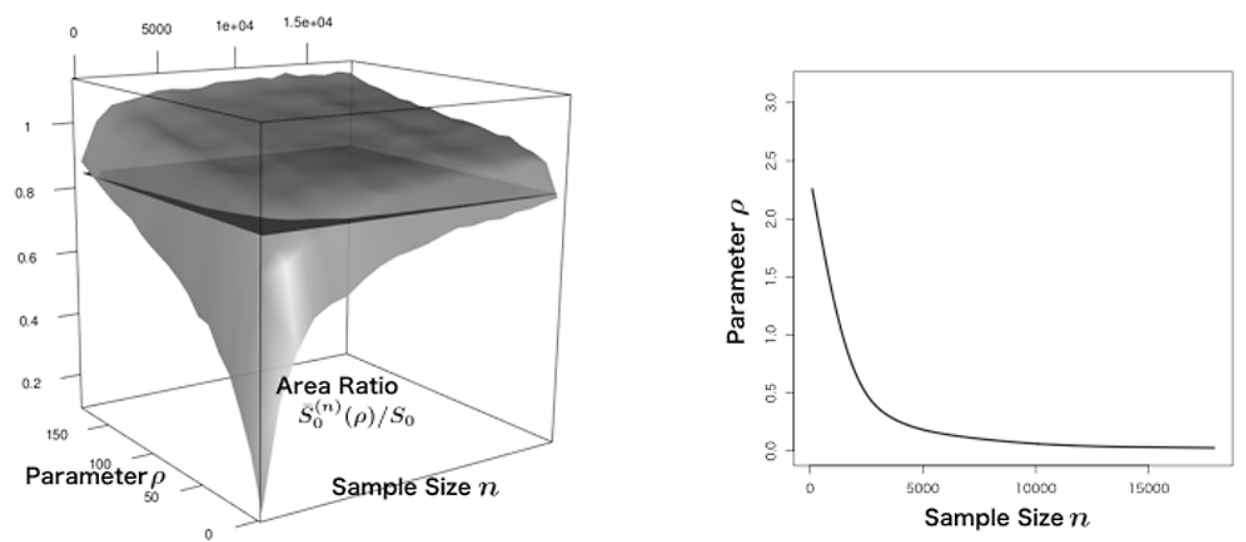

Fig. 5: The 3D plot of the sample size $n$ (x-axis), the parameter $\rho$ ( $y$-axis) and the area ratio $\bar{S}_{0}^{(n)}(\rho) / S_{0}$ ( $z$-axis) (left), and the $\rho$ - $n$ curve (right) in Iwate.

\section{Results}

In the following, as the reconstruction of the extent of damage by the tsunami with the ccc-hull, we show the result at the time of the Great East Japan Earthquake in Iwate. Figure 6 shows the ccc-hull to $n=2000$ CSR points in Iwate. From the 3D plot and the $\rho-n$ curve, the optimal parameter was selected as $\hat{\rho}_{1}=0.033 \pi$ for the sample size $n=2000$. In addition, Figure 7 shows the ccc-hull to $n=2000$ NHPP sample points that is modeled from the elevation in Iwate. From the $3 \mathrm{D}$ plot and the $\rho-n$ curve, the optimal parameter was selected as $\hat{\rho}_{2}=0.2 \pi$ for the sample size $n=2000$. For Figure 6 and 7 , the ccc-hull and Poincaré Cones indicate the estimated safe area and the estimated damage area, respectively. We omitted the output of Poincaré Cones and sample points because it is easy to see. For Figure 6 and 7, we show the extent of damage by the real tsunami like Figure 8. The real damaged area was acquired from Japan Society of Geoinformatics (http://www.jsgi-map.org/tsunami/). In addition, we show agreements of the estimated damage and the real damage by the white area. We can see that the model that considers the elevation indicates better agreement about the estimated damage area and the real damaged area than the one considering CSR points. Table 1 and 2 show the agreement rate of the damaged area or the safe area for CSR and NHPP points, respectively. From pixels for images of simulation results, we calculated the percentage of estimated areas in actual areas 
as the agreement rate. In processing pixels, we used $\mathrm{R}$ package EBImage. For both types of points, the rate that the actual safe area was estimated as being safe is more than $90 \%$. On the other hand, the rate that the actual damaged area was estimated as being damaged is $40 \%$ and $65.2 \%$ for CSR and NHPP points, respectively. Therefore, for our simulation model of the extent of damage by the tsunami with the ccc-hull, to reflect elevations is effective. However, we note that results of Table 1 and 2 will be slightly different each time since locations of points and cones are random. We can see that in the overestimation of the extent of damage by the tsunami in Figure 7. The cause is that sample points for an inland area that the tsunami can't reach are sparse. From the property of the ccc-hull, because the area in which Poincaré Cones can exist increases for sparse sample points, consequently,

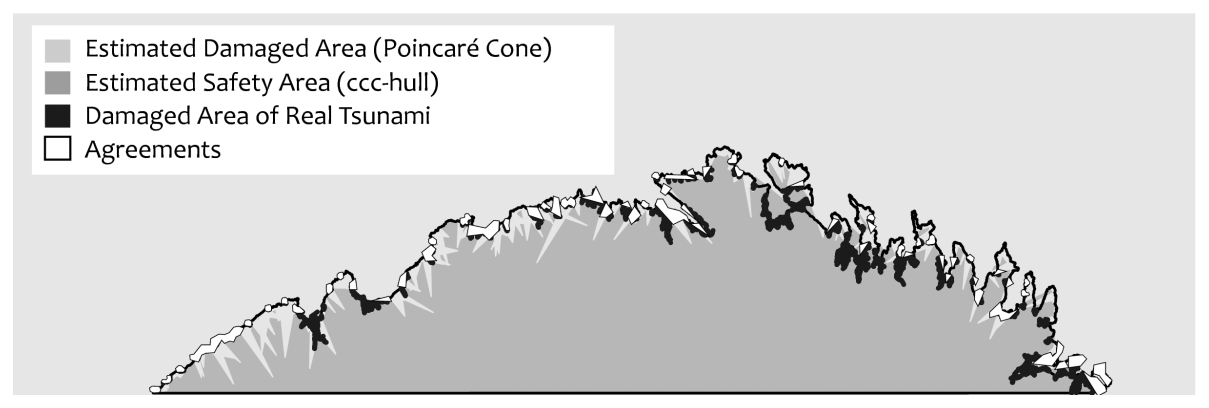

Fig. 6: Simulation of the damaged area in Iwate by the ccc-hull with the optimal parameter $\hat{\rho}_{1}=0.033 \pi$ for $n=2000$ CSR points.

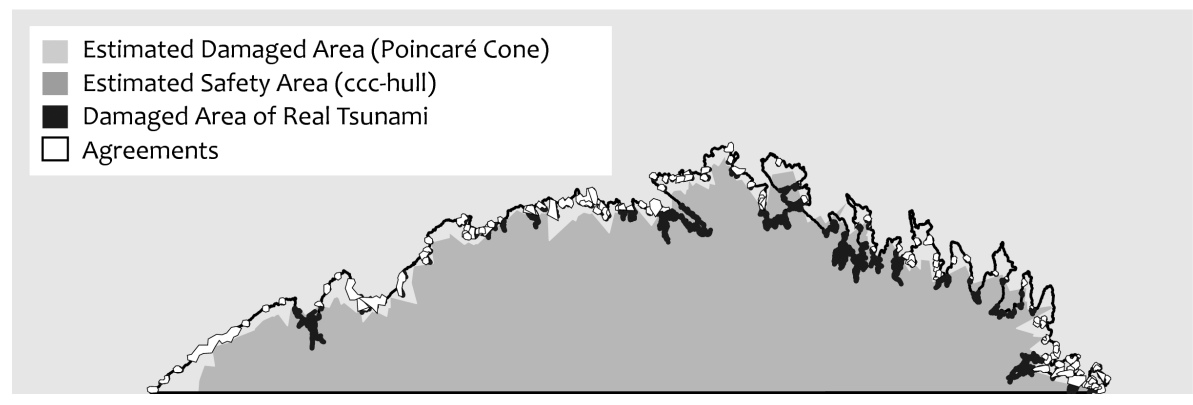

Fig. 7: Simulation of the damaged area in Iwate by the ccc-hull with the optimal parameter $\hat{\rho}_{2}=0.2 \pi$ for $n=2000$ NHPP sample points.

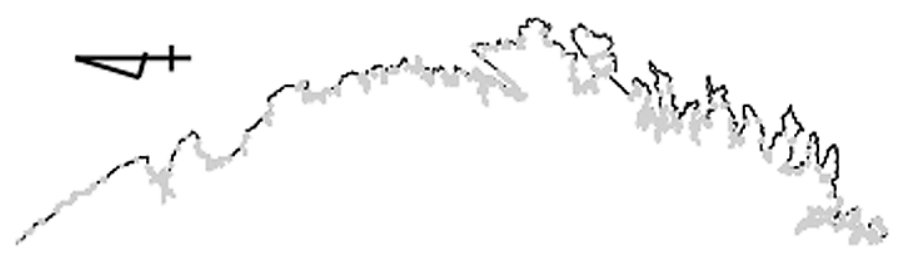

Fig. 8: The real extent of damage by the tsunami at the time of the Great East Japan Earthquake in Iwate. 
Table 1: The agreement rate of the damaged area and the safe area for CSR points.

\begin{tabular}{|c|c|c|}
\hline Type & Estimated damage area (\%) & Estimated safe area (\%) \\
\hline Actual damaged area & 40.0 & 60.0 \\
\hline Actual safe area & 6.7 & 93.3 \\
\hline
\end{tabular}

Table 2: The agreement rate of the damaged area and the safe area for NHPP points.

\begin{tabular}{|c|c|c|}
\hline Type & Estimated damage area (\%) & Estimated safe area (\%) \\
\hline Actual damaged area & 65.2 & 34.8 \\
\hline Actual safe area & 9.9 & 90.1 \\
\hline
\end{tabular}

overestimation occurred. This is because the intensity function that expresses the elevation is not suitable for rejection sampling. Those overestimations will be improved by considering an intensity function such that the difference of elevations declines from inland areas to the coastline.

\section{Conclusion}

In our study, we proposed a simulation model for the extent of damage by a tsunami using the ccc-hull, and reconstructed the damaged area at the time of the Great East Japan Earthquake in Iwate. Then, we modeled the elevation by sampled points from a two-dimensional Non-homogeneous Poisson Process. In addition, we selected the optimal parameter $\rho$ that reconstructed the extent of damage from the $3 \mathrm{D}$ plot, and expressed the intensity of the tsunami by the $\rho-n$ curve. For the result of the reconstruction, using NHPP sample points reecting the elevation gave better agreement of the estimated damage and the real damage than using simple CSR points. Thus, our model that includes the elevation is more effective for the simulation. Previous studies we introduced did not reconstruct the extent of damage by a specific tsunami on the land. For that reason, in this study, we did not compare our new model to the earlier ones.

Our simulation model of the extent of damage by a tsunami with the ccc-hull does not reflect any physical factors other than elevations of the inland areas. To be more precise, we should not ignore elements such as the running direction of the tsunami or the depth of the sea area. In addition, for the tsunami that is expressed by Poincaré Cones, it is difficult to say that the shape of the wave or the damaged area that is seen in two dimensions is correct. The shape of the wave isn't sharp like a cone. However, our model has the advantage, which differentiates it from a conventional one, that it is possible to simulate and visualize the extent of damage by using the ccc-hull with the parameter $\rho$ and the number of sample points $n$ only. In addition, though the visualized extent of damage is broad, it will be possible to apply it to risk perception or the review of evacuation adequately. Therefore, our model with the ccc-hull is effective for the expression of the extent of damage by the tsunami. 


\section{Appendix}

In this section, we show the benchmark result for our improved version. We have used a MacBook Pro with an Intel Core i7 processor $(2.9 \mathrm{GHz})$ and a memory of $8 \mathrm{~GB}$. In addition, for the benchmarking, the $\mathrm{R}$ package rbenchmark was used. We calculated the ccc-hull for CSR points $n=1200$ from a hexagram Figure 9. For the ccc-hull, we have used angle $\rho=\frac{\pi}{4}$ and radius $h=1 / 2$ with $K=300$ cones ( $K$ is the number of cones). The Table 3 shows the benchmark result. The average time that it takes to obtain the hexagram for the above condition once, is 51.050769 seconds for a conventional one and 1.974994 seconds for our algorithm. Therefore, we see that the computational speed is clearly improved by our program.

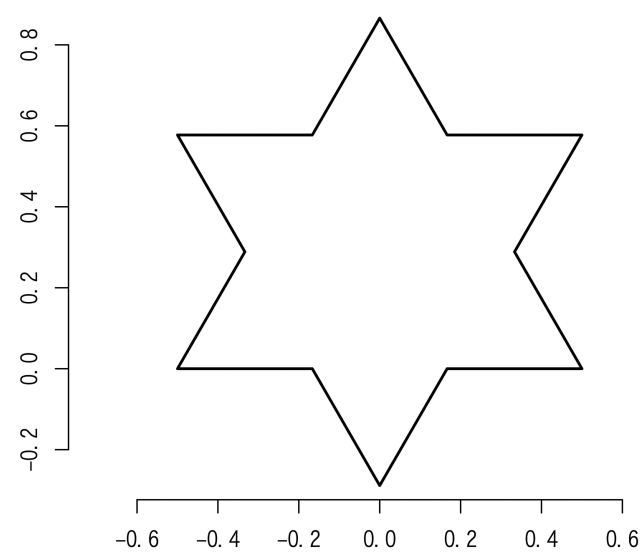

Fig. 9: The domain for the benchmarking (hexagram).

Table 3: The benchmark result.

\begin{tabular}{|c|c|c|c|}
\hline Algorithm & Replications & Elasped (sec) & Average (sec) \\
\hline Cholaquidis & 1000 & 51050.769 & 51.050769 \\
\hline Improved & 1000 & 1974.994 & 1.974994 \\
\hline
\end{tabular}

\section{REFERENCES}

Cholaquidis, A., Cuevas, A. and Fraiman, R. (2014). On Poincaré cone property. Ann. Statist 42, 255-284.

Christian, P., R. and Casella, G. (2009). Introducing Monte Carlo Methods with R. New York: Springer.

Kihara, N., Ota, K., Takabatake, D., Miyagawa, Y. and Matsuyama, M. (2014). Large-scale Experiments of Tsunami Hydrodynamic Load on Vertical Tide Wall. Coast Eng 70(2), 826-830 (in Japanese).

Mörters, P. and Peres, Y. (2010). Brownian Motion. New York: Cambridge University Press. Satake, K. (1991). Recent studies on tsunamis. J. Seism. Soc. Jpn. 44, 99-112 (in Japanese). 
Sato, A. and Sawai, H. (2014). Geographical Risk Assessment from Tsunami Run-up Events based on Socioeconomic-environmental Data and its Application to Japanese Air Transportation. Procedia CIRP 19, 27-32.

Sato, A., Enokitouge, H. and Sawai, H. (2016). Large-scale Computation of Geographical Evaluation Indicators with Socio-economic-Environmental Databases. ISM Research Report 361, 90-101 (in Japanese).

Pateiro-López, B. and Rodríguez-Casal, A. (2010). Generalizing the Convex Hull of a Sample: The R Package alphahull J. Stat. Softw 34, 1-28.

Voss, J. (2013). An Introduction to Statistical Computing: A Simulation-based Approach. New York: Wiley.

Yokota, Y., Ishikawa, T., Watanabe, S., Tashiro, T. and Asada, A. (2016). Seafloor geodetic constraints on interplate coupling of the Nankai Trough megathrust zone. Nature 534, $374-377$.

(Received: March 13, 2016, Accepted: November 21, 2017) 